\title{
Medidas psicológicas en relación con experiencias alucinatorias y experiencias aparicionales
}

Alejandro Parra

Una aparición es una experiencia visual en la que parece estar presente una persona o un animal (vivo o muerto), que está fuera del alcance sensorial de quien lo experimenta. Se pusieron a prueba algunas hipótesis: que la gente que ve apariciones tiene alta capacidad de absorción, propensión a la fantasia, disociación, mayor inestabilidad emocional (neuroticismo) y mayor propensidad a la esquizotipia que los que no las han experimentado. Los resultados mostraron que los individuos que han visto apariciones (u otras experiencias) puntuaron más alto en absorción, disociación, propensidad a la fantasía y esquizotipia cognitivoperceptual que quienes no tuvieron la experiencia. El estudio demostró la viabilidad de adoptar un abordaje psicológico para entender mejor la experiencia de las apariciones.

experiencia alucinatoria / experiencias aparicionales

\section{Psychological measurement of hallucinatory experiences and apparitional experiences}

An apparition is a visual experience in which there appears to be a person or animal present, often in connection with an agent who is dying or undergoing some other crisis. A number of hypotheses were tested: people seeing apparitions and having other apparition-like experiences (experients) have a higher capacity for absorption, fantasy and dissociation proneness, high neuroticism and schizotypy and score higher on three sensory modalities of hallucinatory experience than non-experients. This study demonstrated the viability of adopting a psychological approach in order to better understand the apparitional experience.

hallucinatory experiences / apparitional experiences 


\section{INTRODUCCIÓN}

Thalbourne (1982) definió la aparición como una experiencia visual en la cual parece estar presente una persona o un animal (vivo o muerto) que está fuera del alcance sensorial de quien lo experimenta. Es difícil definir el término 'aparición' sin introducir ciertas presunciones teóricas acerca de la naturaleza de las apariciones. Esta afirmación es más clara si se revisa el estudio llevado a cabo sobre la experiencia de percibir apariciones. La mayor parte de estas investigaciones está determinada por si las apariciones son subjetivas (una creación puramente subjetiva, creada por la mente) u objetivamente reales (que existen de forma independiente de la mente del testigo).

Una revisión de la literatura psicológica sobre alucinaciones revela que solo tres autores (Smythies, 1956; Sarbin \& Juhasz, 1967) tienen cierta familiaridad con la literatura parapsicológica sobre alucinaciones. También hay investigaciones (Bennett, 1987; Davidson \& Russel, 1981; Emmons, 1982; Finucane, 1984) que muestran cómo ha variado la experiencia a lo largo de la historia y en todos los grupos sociales de acuerdo a las funciones sociales de la creencia en apariciones. Sin embargo, no es claro si estas variaciones existen en la experiencia aparicional en sí, o si aparece simplemente en la reconstrucción cognitiva de quien la experimenta o en la descripción narrativa de la experiencia. Gauld (1984) ha examinado casos de experiencias aparicionales anteriores al siglo XVII que están pobremente documentadas y que no pueden considerarse como representativas de la experiencia en sus respectivos períodos históricos. Por lo general, los relatos de experiencias aparicionales provenían de personas que intentaban promover causas filosóficas o teológicas específicas. El abordaje transcultural demuestra cómo las creencias del individuo acerca de la realidad pueden ayudar a determinar si este cree o no que las experiencias particulares son generadas por sí mismos o por otros (Al Issa, 1977, 1995).

Por ejemplo, los análisis cuantitativos ponen en evidencia que la sensación de presencia es común en la experiencia conocida como parálisis del sueño asociada a alucinaciones visuales, auditivas y táctiles, e intenso temor (Cheyne, Rueffer \& Newby-Clark, 1999). La parálisis del sueño es un estado de inmovilidad involuntaria consciente que ocurre antes de dormir o inmediatamente antes de despertar. Un episodio puede durar desde pocos segundos hasta varios minutos, de hecho, aproximadamente el $30 \%$ de adultos jóvenes dijeron haber experimentado parálisis del sueño en tres estudios específicos (Cheyne, NewbyClark \& Rueffer, 1999; Fukuda et al, 1998; Spanos, 1993). Se puede elaborar un escenario complejo, en combinación con experiencias sensoriales particula- 
res, que se identifican como ataques de íncubos, posesiones demoníacas, hechicería o, más recientemente, abducciones alienígenas (Baker, 1990; Cheyne, Newby-Clark \& Rueffer, 1999; Hufford, 1982; Ness, 1978; Spanos, 1993). De este modo, el término alucinación es apropiado porque las experiencias ocurren mientras el individuo está despierto y consciente de lo que lo rodea, y representan la superposición de imaginería onírica que afecta el estado consciente. Por cierto, varias de las características de estas alucinaciones están mapeadas en la fase REM neurofisiológica (Cheyne, Newby-Clark \& Rueffer, 1999). De hecho, el conocido psicólogo norteamericano William James (1902/ 1958, p. 62) definió explícitamente la sensación de presencia como una "alucinación imperfecta".

Hay poca información acerca del perfil de personalidad del individuo que experimenta apariciones. McCreery y Green (1986) encontraron puntajes de psicosis, neurosis y extraversión comparables a los de la población general, pero no hicieron una comparación con individuos sin experiencias aparicionales. Sin embargo, Irwin (1989) observó que la imaginería mental parece estar involucrada en la experiencia. Otros estudios mostraron que individuos con y sin experiencias aparicionales no difieren en su capacidad de absorción psicológica (Houg, 1991), aunque sí una marcada necesidad de procesar sus imágenes mentales (Irwin,
1985), lo cual es consistente con la observación de Palmer (1979) según la cual individuos que tienen experiencias aparicionales analizan sus propios sueños y practican meditación. La propensión a imaginar emerge como un fuerte discriminador: como grupo, estos individuos parecen estar inclinados a fantasear (Cameron \& Roll, 1983; Osis, 1986; Wilson \& Barber, 1983).

Las experiencias aparicionales se presentan con mayor frecuencia en individuos con bajo nivel educativo, y en personas que enviudaron recientemente la sensación de presencia es relativamente común. Palmer (1979) no encontró ninguna correlación con género, raza, edad o religiosidad. En la primera colección de casos de los fundadores de la Society for Psychical Research de Londres (Gurney, Myers \& Podmore, 1886) y en el "Censo de Alucinaciones" (Sidgwick, Johnson, Myers, Podmore \& Sidgwick, 1894) se recogió el testimonio de 17.000 personas, de las cuales aproximadamente el $10 \%$ tuvo alguna clase de experiencia alucinatoria. Había 830 relatos de apariciones que tenían forma humana y aparentaban ser bastante reales. La teoría tradicional de la aparición favorece la hipótesis de la intervervención de espíritu, es decir, que una aparición es un aspecto de la existencia humana que sobrevive a la muerte del cuerpo. Pero los datos del censo no encajaban bien con esta teoría; por ejemplo, algu- 
nos eran de animales y no de seres humanos, y solo el 20\% (de 830 casos) era de personas muertas (fantasmas). Este aspecto de alucinación benigna (de gente sana) es importante porque estas alucinaciones difieren de las que se observan en la enfermedad mental o por efectos de las drogas (Anderson \& Anderson, 1982; Asaad \& Shapiro, 1986).

A causa de la dificultad de decidir entre teorías subjetivas y objetivas, lo más interesante es la experiencia de la aparición en sí misma. Green y McCreery (1975) intentaron mapear las dimensiones de la experiencia aparicional. Por ejemplo, Osis (1986) definió la experiencia aparicional en vez de la aparición, si bien Osis y MacKenzie (1982) no aceptaban la palabra alucinación como un rótulo apropiado para quien experimenta una aparición. En tal sentido, no hay nada en las características nucleares del estudio de Osis que haga pensar en algo más allá de una experiencia subjetiva. Por ejemplo, el famoso psicopatólogo alemán Kurt Schneider (1959) consideraba las alucinaciones auditivas (oír voces) como el principal síntoma de la esquizofrenia. Los estudios más recientes han demostrado que las alucinaciones, sobre todo las de modalidad auditiva, aparecen en la mayoría de los pacientes diagnosticados con esquizofrenia (Sartorius, Shapiro \& Jablensky, 1974). Sin embargo, un sorprendente número de individuos normales, cuando se les pre- gunta, dicen haber experimentado una historia de experiencias alucinatorias (Bentall \& Slade, 1985; Posey \& Losch, 1983; Tien, 1991), e incluso hay una fuerte evidencia de la existencia de variaciones culturales en la predisposición a tener las experiencias alucinatorias (Al Issa, 1977, 1995).

Existen tres observaciones que han permitido a los investigadores entender los procesos cognitivos involucrados en las alucinaciones auditivas. Primero, hay evidencia de que las experiencias alucinatorias frecuentemente ocurren más en períodos de tensión, o cuando el individuo está expuesto a un entorno de estímulos o por la falta de estos, o en extremo ruidoso (Slade \& Bentall, 1988). Segundo, las alucinaciones auditivas están acompañadas por pequeños movimientos de los músculos vocales y la activación de áreas del discurso del hemisferio izquierdo del cerebro. Muchos investigadores han concluido que deben tener en cuenta estas hipótesis suponiendo que las alucinaciones auditivas se atribuyan al discurso interno (Bentall, 1990; Hoffman, 1986), es decir, las personas que oyen voces parecen estar hablando con sus alucinaciones de una manera relativamente normal, aunque lo verdaderamente anormal es que crean que su voz interna proviene de alguien externo.

Otros estudios también han demostrado (Mavromatis, 1987; Sherwood, 1999) que las apariciones ocurren a menudo en estados de conciencia en 
los cuales las imágenes mentales tienen la particularidad de parecer vivas y reales (por ejemplo en los estados hipnagógico/hipnopómpico). Los pacientes psicóticos con alucinaciones auditivas difieren de los pacientes que no alucinan, y de las personas normales al tener un "testeo de realidad" debilitado (Slade, 1976; Johnson \& Raye, 1981). El testeo de realidad se refiere a la habilidad de distinguir una percepción actual (realidad) de un acto de imaginación actual (alucinación o aparición). El testeo de realidad también es importante para las personas normales que tienen alucinaciones visuales o apariciones, aunque los objetos son recuerdos evocados por la memoria de una aparición vista en el pasado, es decir que se refiere a la distinción entre una percepción pasada y un acto de imaginación pasado. En conclusión, el testeo de realidad es importante, pero un aspecto de este es la habilidad de focalizar la atención (absorción), y el otro es la habilidad de crear historias por fuera de la imaginería visual (propensión a la fantasía) del individuo.

Este estudio apunta a analizar si las experiencias aparicionales, la sensación de presencia - el cual se refiere a la vívida sensación de alguien o algo presente en un lugar (por ejemplo una habitación) donde en realidad no hay nadie (Cheyne, Newby-Clark \& Rueffer, 1999)- y la experiencia espontánea de mediumnidad/posesión -que se refiere a casos en los que el cuerpo de una persona es tomado por otra personalidad o entidad- como fenómeno digno de estudio, además de otros aspectos de experiencia humana. De este modo, el enfoque está en la experiencia o fenómeno, cualquiera que sea su interpretación, en lugar de la aparición en sí misma. Las apariciones, como todo en la vida mental de un individuo saludable, no ocurren en un vacío, sino que se interconecta con muchos otros procesos psicológicos.

Por esta razón, considero que los casos de apariciones son parte de la experiencia humana, y como tal merecen ser comprendidos y estudiados. Se conoce poco de los factores que subyacen en las variables psicológicas y los procesos que pueden influir en las experiencias aparicionales, pero en la literatura psicológica, parapsicológica y psiquiátrica hay indicadores de que algunas variables cognitivas (mentales) particulares son importantes. Analizaremos aquí tres variables cognitivas: la disociación y la absorción, la propensión a la esquizotipia, la inestabilidad emocional (neuroticismo) y la propensión a la fantasía.

\section{HIPÓTESIS}

Este estudio - de carácter exploratoriopone a prueba seis hipótesis específicas: que personas que ven apariciones o que tienen otras experiencias cuasi aparicionales tienen mayor 1) absorción, 2) propensidad a la fantasía, 3) 
tendencia a la disociación, 4) inestabilidad emocional (o emocionalidad negativa), 5) propensidad a la esquizotipia cognitivo-perceptual, interpersonal y desorganizada, y 6) experiencia alucinatoria auditiva, visual y táctil que quienes no las tienen. Se llevará a cabo un análisis post hoc con algunos de los ítems de la CEA.

\section{MÉtodo}

\section{Participantes}

La muestra incluyó 656 estudiantes de psicología de ambos sexos, 494 (76\%) mujeres y $154(24 \%)$ varones, con un rango etario de 17 y 57 años (Media $=$ 25.11; $\mathrm{SD}=7.23$ ) residentes en la ciudad de Buenos Aires y alrededores. Ningun estudiante recibió compensación económica.

\section{Instrumentos}

Los estudiantes completaron seis escalas, cinco de estas de experiencias perceptuales: la Escala de Imaginería (QMI) (Sheehan, 1967; Richardson, 1990; alfa de Cronbach $=.77$ ) un inventario de 35 ítems que mide la intensidad de la imaginería en una escala Likert 1-7, siendo 1 alta imaginería y 7 baja imaginería, en siete modalidades sensoriales (solo dos se usaron: visual, por ejemplo: "El sol poniéndose en el horizonte", y cinética, por ejemplo: "Alcanzar un estante alto"), el Cuestionario de Alucinaciones (CEA) (Parra,
Adróver \& González, 2006; alfa de Cronbach $=.93$ ) que mide la propensidad a alucinar en seis modalidades sensoriales de 38 ítems con una escala Likert 0 - 5, siendo 1 rara vez y 5 frecuentemente (solo dos se usaron: visual, por ejemplo: "He visto sombras, o figuras humanas o no humanas cerca de mi cama, yo las he visto claramente y veo lo que hacen"; y táctiles, por ejemplo: "He tenido la experiencia de sentir una palmada en mi hombro, o cualquier otra sensación vívida de contacto fisico de otra persona detrás de mí, pero cuando me doy vuelta no veo a nadie"), la Escala de Experiencias Disociativas (DES) (Bernstein y Putnam, 1993; alfa de Cronbach = .91) una escala trifactorial de 28 ítems en una escala Likert 0 - 10 que mide una variedad de tendencias disociativas: amnesia, despersonalización y desrealización (por ejemplo: "Algunas personas tienen la experiencia de conducir o estar viajando en un coche, colectivo o tren subterráneo y de repente se dan cuenta de que no recuerdan lo que pasó durante todo o parte del viaje"), y el Cuestionario de Experiencias Creativas (CEQ) (Merckelbach, Horselenberg \& Muris, 2001; alfa de Cronbach $=.89$ ), una escala de 25 ítems de respuesta verdadero/falso que mide la propensidad a la fantasía, especialmente la tendencia a fantasear en la niñez (por ejemplo, "Cuando veo escenas de violencia por televisión, me siento tan involucrado en estas que me provoca 
mucha inquietud"), y la Escala de Absorción de Tellegen (TAS) (Tellegen y Atkinson, 1974; alfa de Cronbach = .91) que mide en 34 ítems de respuesta verdadero/falso la frecuencia con que una persona se involucra en actividades que exigen atención y concentración (por ejemplo: "Cuando escucho música de órgano u otra música imponente a veces siento como si me estuvieran levantando en el aire").

Se empleó también una medida de rasgos de personalidad el Cuestionario de Personalidad Esquizotípica (SPQ) (Raine, 1991, 1992; Raine \& Baker, 1992; Raine \& Benishay, 1995; alfa de Cronbach $=.91$ ) una escala trifactorial de 74 ítems de respuesta dicotómica si/no que mide estilos de pensamiento esquizotípicos: factor cognitivo perceptual (por ejemplo: "¿Alguna vez ha visto cosas que para los demás son invisibles?" o "¿Son sus pensamientos a veces tan fuertes que usted casi podría escucharlos?"), interpersonal (por ejemplo: "Tengo poco interés en conocer a otras personas" o "Soy muy pobre al expresar mis verdaderos sentimientos por el modo en el que hablo y miro"), y desorganizado (por ejemplo "Otras personas me ven como desatento o excentrico" o "A veces utilizo palabras de un modo inusual"). El Inventario de Personalidad de Eysenck (Eysenck \& Eysenck, 1978) es una escala dimensional de personalidad ampliamente usada que mide neuroticismo, extroversión y psicoticismo. En general, un valor alfa de Cronbach mayor a .60 es una medida aceptable de confiabilidad (Grady \& Wallston, 1988; Kim \& Mueller, 1978). Las medidas de alfa corresponden a la versión en español de cada escala.

Respecto a las experiencias aparicionales, la sensación de presencia y la mediumnidad/posesión se formularon preguntas (basadas en la encuesta de Palmer, 1979). Para experiencia aparicional (EA): "Estando despierto, he tenido la experiencia de oír voces o ver presencias invisibles para otros que me indicaban acerca de un peligro inminente que luego ocurrió"; para sensación de presencia (SP): "Estando solo he tenido la vívida impresión de una sensación de presencia, pero no visible donde me encontraba"; para mediumnidad/posesión (MP): "He tenido la experiencia de sentir que mi cuerpo no me pertenecía y que estaba siendo controlado por otra fuerza aparte de mí (si duda, por favor responda no)". Si la respuesta es afirmativa, la pregunta también mapea tres dimensiones de la experiencia: frecuencia de la experiencia, explicación subjetiva (racional, deconocida y paranormal) de la experiencia, y positivo o negativo impacto (emocional) en una escala Likert, donde 1 es positivo y 7 negativo.

\section{Procedimiento}

Aplicamos una técnica de muestreo no probabilística para obtener el mayor número de casos para analizar. El set 
de tests autoadministrables fue entregado en un sobre A4 a cada estudiante, en forma contrabalanceada, durante una clase teórica. El tiempo promedio para completar los cuestionarios fue de 50 minutos. Los estudiantes recibieron una vaga información del objeto de estudio y se les invitó a participar voluntaria y anónimamente completando los test, en una única sesión, en días y horarios previamente pactados con los docentes. El orden de administración de ambas pruebas fue contrabalanceado y los cuestionarios de alucinaciones se presentaron bajo el seudotítulo de Cuestionario de Experiencias Psicológicas, con lo cual se evitó sesgar las respuestas. Los análisis se procesaron mediante el SPSS 11.5 (en español) y los análisis estadísticos fueron evaluados a dos colas.

\section{Resultados}

Sobre la base de las respuestas obtenidas para las preguntas de EA, SP y MP se formaron dos grupos para cada experiencia. Para agrupar a quienes tenían EA, SP y MP o experientes se convirtieron las respuestas 1 (rara vez), 2 (ocasionalmente), 3 (a menudo) y 4 (muy frecuentemente). Para agrupar a quienes no tuvieron EFC los (no experientes) se empleó solo la respuesta "Nunca".

\section{Resultado de las hipótesis}

Absorción, propensión a la fantasía y disociación. La hipótesis 1 consistía en que los estudiantes con experiencias puntuarían más alto que quienes no tuvieron experiencia en absorción (medido con el TAS), la cual fue confirmada: quienes tienen experiencias aparicionales puntuaron significativamente más alto que quienes no la tuvieron (EA: $\mathrm{z}=6.06, \mathrm{p}<.001$, a dos colas; SP: $z=5.19, \mathrm{p}<.001$, a dos colas y MP: $\mathrm{z}=2.11, \mathrm{p}<.05$, a dos cola s). La hipótesis 2 planteaba que los estudiantes con experiencias puntuarían más alto que quienes no la tuvieron en propensión a la fantasía (medido con el CEQ), la cual fue confirmada: quienes tenían experiencias aparicionales puntuaron significativamente más alto que quienes no la tuvieron (EA: $\mathrm{z}=4.34, \mathrm{p}<.001$, a dos colas; SP: $\mathrm{z}=5.17, \mathrm{p}<.001$, a dos colas y MP: $\mathrm{z}=2.12, \mathrm{p}<.05$, a dos colas). La hipótesis 3 era que los estudiantes con experiencias puntuarían más alto que quienes no tuvieron la experiencia en disociación (medido con el DES), la cual fue solamente confirmada para EA $(z=4.65, p<.001$, a dos colas) y SP $(\mathrm{z}=4.95, \mathrm{p}<.001$, a dos colas), pero no para MP, la cual no fue significativa (véase tabla 3 ). 
Tabla 1

Experiencia aparicional, sensación de presencia y experiencias de mediumnidad/posesión: frecuencia, explicación e impacto emocional

\begin{tabular}{|c|c|c|c|c|}
\hline & & $\begin{array}{l}\text { Experiencia } \\
\text { aparicional }\end{array}$ & $\begin{array}{l}\text { Sensación de } \\
\text { presencia }\end{array}$ & $\begin{array}{l}\text { Mediumnidad/ } \\
\text { posesión }\end{array}$ \\
\hline & $\begin{array}{l}\text { Sin experiencia } \\
\text { Con experiencia }\end{array}$ & $\begin{array}{c}N=582(89.68) \\
N=67(10.32)\end{array}$ & $\begin{array}{l}N=354(54.55) \\
N=295(45.45)\end{array}$ & $\begin{array}{c}N=594(91.53) \\
N=55(8.47)\end{array}$ \\
\hline $\begin{array}{l}\text { Con } \\
\text { experiencia }\end{array}$ & $\begin{array}{l}\text { Género } \\
\text { Rango de edad } \\
\text { Media de edad - SD }\end{array}$ & $\begin{array}{c}M=13(19.4) \\
F=54(80.6) \\
18-57 y r . \\
24.72-7.21\end{array}$ & $\begin{array}{c}M=66(22.4) \\
F=229(77.6) \\
17-57 \text { yr. } \\
25.35-7.36\end{array}$ & $\begin{array}{c}M=1(20.0) \\
F=44(80.0) \\
17-57 y r . \\
25.69-7.37\end{array}$ \\
\hline $\begin{array}{l}\text { Sin } \\
\text { experiencias }\end{array}$ & $\begin{array}{l}\text { Género } \\
\mathrm{F}=439(75.4) \\
\text { Rango de edad } \\
\text { Edad media-SD }\end{array}$ & $\begin{array}{c}M=143(24.6) \\
F=268(74.9) \\
17-54 y r \\
25.56-7.14\end{array}$ & $\begin{array}{c}\mathrm{M}=90(25.1) \\
\mathrm{F}=449(76.1) \\
17-54 \mathrm{yr} . \\
25.67-7.08\end{array}$ & $\begin{array}{c}M=141(23.9) \\
18-57 y r . \\
25.69-7.37\end{array}$ \\
\hline
\end{tabular}

Tabla 2

Frecuencia, impacto emocional y explicación de la experiencia aparicional, sensación de presencia y experiencias de mediumnidad/posesión

\begin{tabular}{llccc}
\hline & & $\begin{array}{c}\text { Experiencia } \\
\text { aparicional } \\
(\mathbf{N}=\mathbf{6 7})\end{array}$ & $\begin{array}{c}\text { Sensación de } \\
\text { presencia } \\
(\mathbf{N}=\mathbf{2 9 5})\end{array}$ & $\begin{array}{c}\text { Mediumnidad/ } \\
\text { posesión } \\
\mathbf{( N = 5 5 )}\end{array}$ \\
\hline Frecuencia & A veces & $36(53.7)$ & $79(26.8)$ & $24(43.6)$ \\
& Frecuentemente & $29(43.3)$ & $194(65.8)$ & $29(52.8)$ \\
Impacto & Racional/Explicable & $4.15-2.09$ & $3.95-1.86$ & $2(3.6)$ \\
emocional & Desconozco & $10(14.9)$ & $69(23.4)$ & $21(38.2)$ \\
Explicación & Paranormal/Inexplicable & $29(43.3)$ & $155(52.5)$ & $23(41.8)$ \\
& Media $(1-7)(1)-S D$ & $28(41.8)$ & $71(24.1)$ & $11(20.0)$ \\
\hline
\end{tabular}

(1) 1 negativo a 7 positivo para el impacto emocional. 


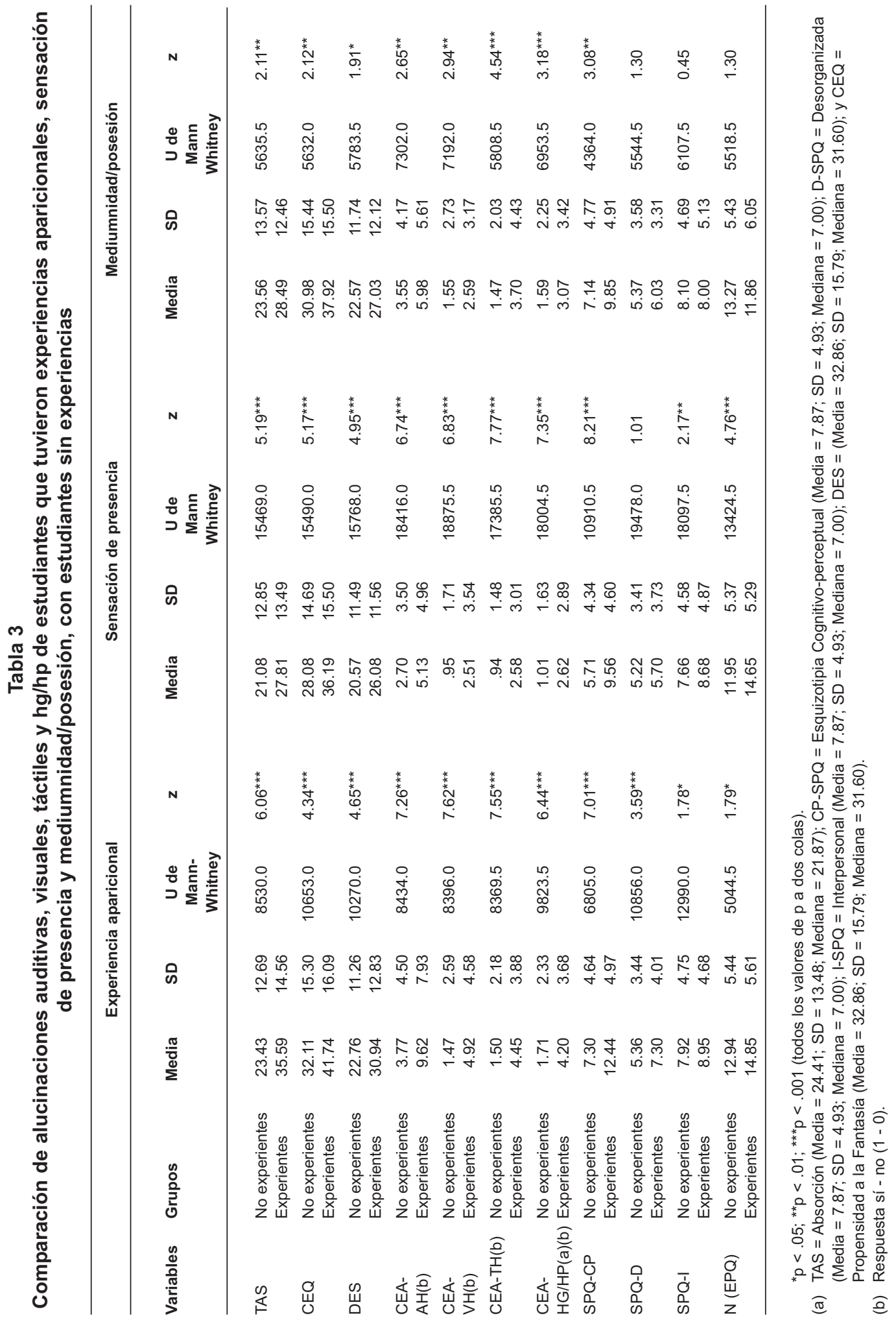


Propensión de esquizotipia y neuroticismo. La hipótesis 4 se refiere a que los estudiantes con experiencias puntuarían más alto que quienes no tuvieron experiencias en neuroticismo (medido con el EPQ), la cual fue confirmada solo para SP $(z=4.76, p<.001$, a dos colas), pero no fue significativa para EA y MP. La hipótesis 5 dice que los estudiantes con experiencias puntuarían más alto que quienes no tuvieron experiencias en esquizotipia cognitivo-perceptual (EA: $\mathrm{z}=7.01, \mathrm{p}<.001$, a dos colas; SP: $\mathrm{z}=8.21, \mathrm{p}<.001$, a dos colas y MP: $\mathrm{z}=3.08, \mathrm{p}<.05$, a dos colas) (medido con el SPQ). Sin embargo, los factores de esquizotipia interpersonal y desorganizada no fueron significativos (excepto solo desorganizada para AP: $\mathrm{z}=3.59, \mathrm{p}<001$, a dos colas) (véase tabla 3 ).

Experiencias alucinatorias. La hipótesis señalaba que los estudiantes con experiencias alucinatorias puntuarían más alto en disociación que quienes no tuvieron experiencias, la cual se confirmó para alucinación de modalidad auditiva (oír voces) (EA: $\mathrm{z}=7.26$, $\mathrm{p}<.001$, a dos colas; SP: $\mathrm{z}=6.74$, $\mathrm{p}<.001$, a dos colas y SM: $\mathrm{z}=2.65$, $\mathrm{p}=.008$, a dos colas), visual (EA: $\mathrm{z}=7.62, \mathrm{p}<.001, \mathrm{a}$ dos colas; SP: $\mathrm{z}=6.83, \mathrm{p}<.001$, a dos colas y MP: $\mathrm{z}=2.94, \mathrm{p}=.003, \mathrm{a}$ dos colas), táctil (EA: $\mathrm{z}=7.55, \mathrm{p}<.001$, a dos colas; SP: $z=7.77, p<.001$, a dos colas $y$ SM: $\mathrm{z}=4.54, \mathrm{p}<.001$, a dos colas), $\mathrm{y}$ la modalidad alucinatoria hipnagógi- co/hipnopómpica (EA: $\mathrm{z}=6.44$, $\mathrm{p}<.001$, a dos colas; SP: $\mathrm{z}=7.35$, $\mathrm{p}<.001$, a dos colas y MP: $\mathrm{z}=3.18$, $\mathrm{p}=.001$, a dos colas), donde la puntuación para los estudiantes era significativamente más alta que para quienes no tenían la experiencia (véase tabla 3).

También se analizaron las tres variables juntas: absorción, disociación y propensión a la fantasía, las cuales mostraron una diferencia significativa entre ambos grupos (con y sin experiencia). Las puntuaciones del TAS, DES y CEQ de los estudiantes que tenían experiencias eran significativamente más altas que los que no las tuvieron, indicando significativamente más experiencias (medidas con el EPQ) en relación con experiencias alucinatorias. El grado de espiritualidad $(1=$ alta espiritualidad y 5 = baja espiritualidad) tomado también como variable, mostró una diferencia significativa entre ambos grupos (con y sin experiencia), indicando que de alguna forma la espiritualidad era significativamente más alta para quienes decían haber tenido impresiones vívidas o sensaciones de una presencia invisible $(\mathrm{z}=3,63, \mathrm{p}<.001$, a dos colas).

\section{Análisis de regresión logistica}

¿Cuál de las once variables discriminan mejor entre experientes de apariciones y no experientes? Se llevó a cabo un análisis de regresión logística binaria para buscar el principal predictor. Parcialmente debido a un problema 
de colinealidad, después de verificar los requerimientos de la técnica, se llevó a cabo un análisis por el método de pasos hacia delante de Wald. Para reducir la colinealidad se excluyeron del análisis de regresión solamente algunas variables, como neuroticismo y esquizotipia interpersonal.

Experiencia aparicional. De una muestra de 543 participantes, los resultados del mejor modelo (paso 2) mostró que esquizotipia cognitivo-perceptual era el mejor predictor para la experiencia aparicional $(\mathrm{si} / \mathrm{no})[\beta=$ .16 , Wald $=25.20 ; \mathrm{gl}=1 ; \mathrm{p}<.001$; $\operatorname{Exp}(B)=1.18 ; R^{2}$ de Nagelkerke's $=$ .19], y en segundo orden absorción como predictora $[\beta=.03$, Wald $=9.41$; $\mathrm{gl}=1 ; \mathrm{p}=.002 ; \operatorname{Exp}(\mathrm{B})=1.03 ; \mathrm{R}^{2} \mathrm{de}$ Nagelkerke $=.22]$, pero ambas en un grado débil. Esto sugiere que la absorción puede estar por debajo de la diferencia entre ambos grupos, mientras que el resto de las variables no resultaron predictoras. Sin embargo, la experiencia aparicional parece ser una variable continua en el sentido de que la experiencia puede ocurrir más de una vez. Un análisis de la frecuencia de impacto (positivo) emocional (Media $=$ 4.15; $\mathrm{SD}=2.09$, donde 1 es positiva a 5 negativa) no resultó significativa.

Sensación de presencia. De una muestra de 399 participantes, los resultados del mejor modelo (paso 1) mostró que esquizotipia cognitivo-perceptual era el mejor predictor para la experiencia aparicional (sí/no) $[\beta=.18$, Wald $=$
51.44; $\mathrm{gl}=1 ; \mathrm{p}<.001 ; \operatorname{Exp}(\mathrm{B})=1.19 ;$ $\mathrm{R}^{2}$ de Nagelkerke's $=.19$ ], en un grado moderadamente intenso. Esto sugiere que la modalidad cognitivo-perceptual puede distinguir entre ambos grupos (experientes vs. no experientes), mientras que el resto de las variables no resultaron predictoras. Sin embargo, la experiencia aparicional parece ser una variable continua en el sentido de que la experiencia puede ocurrir más de una vez. Un análisis de la frecuencia de impacto (negativo) emocional (Media $=$ 4.44; $\mathrm{SD}=2.21$, siendo 1 positiva a 5 negativa) no resultó significativa.

\section{Discusión}

Las características de las apariciones y las experiencias cuasi aparicionales (por ejemplo, la mediumnidad/posesión y la sensación de presencia) en este estudio es similar al de estudios anteriores, y es consistente con las características descritas por Osis (1986). Además, el concepto de monitoreo de realidad puede ser crucial para comprender mejor la experiencia aparicional, el cual no requiere que el individuo que tiene la experiencia sea particularmente hábil para producir imágenes visuales vívidas.

El estado de absorción está asociado con un bajo nivel de testeo de realidad. Durante este estado, el objeto focal de atención, aunque es imaginario, es completamente real para el individuo (Tellegen y Atkinson, 1974). Quizá la capacidad de absorción es la 
única de una constelación de factores relacionados, y el estilo puede ser más importante que la capacidad o habilidad. O, quizá debemos distinguir entre dos tipos de testeo de realidad: la atención momentánea y la mayor habilidad para suspender el testeo de realidad. El TAS mide solo la capacidad de absorción -el grado en que una persona está concentrada o inmersa en una experiencia mental en un momento dadode modo que el testeo de realidad está temporalmente inhibido. Una escala que mida la necesidad de absorción (una variable motivacional, véase Irwin, 1985), puede indicar una forma más regular o un deseo recurrente de estar inmerso en una actividad mental, ya que el testeo de realidad -que funciona debilitado- se transforma en un aspecto estable del estilo cognitivo.

Wilson y Barber (1983) acuñaron el término de "personalidad con propensión a la fantasía" para referirse a un pequeño grupo de personas, aproximadamente el $4 \%$ de la población, que fantasea la mayor parte del tiempo. Pueden ver, oir y tocar absolutamente todo lo que fantasean. Su imaginería eidética es el extremo de la habilidad para visualizar vívidamente, y se caracteriza por una profunda absorción en su fantasía, es decir, una capacidad habitual de suspender su monitoreo de realidad, diferente a su capacidad de absorción. Las personas estudiadas por Wilson y Barber tenían una variada y amplia gama de experiencias psíquicas, incluyendo las apariciones. Un problema es que algunos solo tenían fantasía visual con los ojos cerrados, de modo que no podían ver apariciones -que por definición requieren que los ojos de la persona estén abiertos-. En dos estudios (Council y Huff, 1990; Myers y Austrin, 1985), se encontró una fuerte correlación entre la propensión a la fantasía y la absorción, y ambos correlacionaron significativamente con experiencias psíquicas, particularmente la percepción (visual, auditiva o táctil) de apariciones.

Se ha empleado el concepto de propensión a la fantasía porque quienes tuvieron la experiencia aparicional son claramente más propensos a fantasear que quienes no la tuvieron. Esto no significa necesariamente que todas las apariciones sean pura fantasía, ya que algunas parecen ser verídicas. En otras palabras, la información extrasensorial está incorporada en la fantasía, pero a causa de una debilidad en el testeo de realidad, los individuos experimentan la aparición como una imagen alucinatoria con intenso contenido de realidad. De modo que en el contexto de este estudio, la distinción entre experiencias completamente subjetivas y las consideradas paranormales (verídicas) es irrelevante. Incluso las experiencias verídicas pueden depender del mismo factor psicológico de predisposición, como ocurre con las experiencias aparicionales subjetivas. 
Estos resultados también sugieren que las visiones de fantasmas se pueden relacionar con procesos cognitivos que involucran a la propensión a la fantasía y la propensión a la esquizotipia cognitiva-perceptual, y que estos factores están correlacionados entre sí. En primer lugar, se puede concluir que las experiencias aparicionales y cuasi-aparicionales están relacionadas a niveles más altos de absorción, disociación y experiencias de fantasía/imaginación, lo cual coincide conceptualmente con los estudios que han encontrado que la propensión a la fantasía parece ser un predictor funcional de los fenómenos parapsicológicos (Myers, Austrin, Grisso \& Nickeson, 1983; Wilson \& Barber, 1983). La absorción es la capacidad de centrar la atención exclusivamente en algún objeto (incluyendo la imaginería mental), que parecen tener una elevada percepción de realidad, como ocurre con las apariciones (MacKenzie, 1982). La capacidad de absorción por sí misma puede no ser suficiente; quizás las personas también deben estar motivadas o necesitar la experiencia de absorción, así como una situación apropiada para inducirlo. Un nivel alto de absorción indica la habilidad de inhibir momentáneamente el testeo de realidad, y de hecho las personas que puntúan alto en absorción, también tenían alta incidencia de experiencias paranormales subjetivas, incluyendo las apariciones (véase Irwin, 1985).
En segundo lugar, aunque hay una clara superposición entre las creencias paranormales, las experiencias paranormales y la esquizotipia, no necesariamente significa que las creencias y las experiencias paranormales estén asociadas al malestar psicológico (McCreery \& Claridge, 1995, 1986; McCreery, 2006). Por ejemplo, individuos con experiencias fuera del cuerpo, pero que no mostraban ningún síntoma negativo, tenían sin embargo mayores síntomas positivos de esquizotipia que quienes no tenían tales experiencias. Incluso, algunas de estas personas parecían no solo ser saludables a pesar de sus experiencias, sino gracias a estas. McCreery y Claridge (2002, p. 144) denominaron al conjunto de estos síntomas positivos como esquizotipia feliz, el cual representa un modelo cuasi dimensional de esquizotipia que sugiere una extensión del modelo dimensional con la salud como punto de partida (Claridge, 1997; Claridge \& Beech, 1995).

Los individuos que tienen experiencias aparicionales también indican poder recordar sueños frecuentemente, experiencias místicas y experiencias fuera del cuerpo (Gómez Montanelli \& Parra, 2005), en asociación con disociación y susceptibilidad hipnótica (Pekala, Kumar \& Marcano, 1995; Parra \& Argibay, 2006), lo cual también se corresponde con los resultados de Kohr (1980) y Palmer (1979) que relacionan las apariciones con otras expe- 
riencias, es decir, es raro encontrar a una persona que dice tener experiencias de apariciones pero que no tiene ninguna otra experiencia paranormal. Sin embargo, debemos enfatizar que estamos tratando con afirmaciones que dependen solamente de respuestas de encuestas. El significado de estas afirmaciones no es claro si no estamos seguros de que las respuestas de los participantes están relacionadas con lo que preguntamos; debemos convencernos de que no podemos esperar tener mayor información sin llevar a cabo entrevistas de seguimiento o, al menos, tener una descripción por escrito de las experiencias.

Sin embargo, como piensan Johnson, Hashtroudi y Lindsay:

[...] es más probable que un individuo integrado en un entorno que admite la existencia de fantasmas o que valora las experiencias espirituales le atribuya contenido de realidad a la imagen de un pariente fallecido, que uno integrado en un entorno materialista y científico [...]. A un individuo le será más fácil adoptar un criterio liberal al asumir el carácter realístico de los eventos que percibe en condiciones de estimulación externa minimizada, a consecuencia de lo cual será más probable que malinterprete la ideación interna a una fuente externa (1993: 26).

Esto confirma que las experiencias aparicionales (y cuasiaparicionales) pueden tener importantes implicaciones para la clínica. Muchos terapeutas todavía consideran a su paciente, o a quien dice tener experiencias de apari- ciones ( $u$ otras experiencias parapsicológicas), como mentalmente enfermo o delirante (Tart, 1983a, 1983b, 1984, Gómez Montanelli \& Parra, 2003). Por esta razón, las personas propensas a la fantasía, temiendo al ridículo, a menudo no comentan a nadie sobre tales experiencias.

\section{REFERENCIAS}

Al-Issa, I. (1977). Social and cultural aspects of hallucinations. Psychological Bulletin, 84, 570-587.

AI-Issa, I. (1995). The illusion of reality or the reality of an illusion: Hallucinations and culture. British Journal of Psychiatry, 166, 368-373.

Anderson, R. \& Anderson, W. (1982). Veridical and psychopathic hallucinations: A comparison of types. Parapsychology Review, 13(3), 17-23.

Asaad, G. \& Shapiro, B. (1986). Hallucinations: Theoretical and clinical overview. American Journal of Psychiatry, 143, 1088-1097.

Baker, R.A. (1990). They call it hypnosis. Búfalo, Nueva York: Prometheus.

Bennett, G. (1987). Traditions of belief: Women and the supernatural. Londres: Penguin Books.

Bentall, R. P. (1990). The illusion of reality: A review and integration of psychological research on hallucinations. Psychological Bulletin, 107, 82-95. 
Bentall, R. P. \& Slade, P. D. (1985). Reality testing and auditory hallucinations: A signal detection analysis. British Journal of Clinical Psychology, 24, 159-169.

Bernstein, E. M. \& Putnam, F.W. (1986). Development, reliability, and validity of a dissociation scale. Journal of Nervous and Mental Disease, 174, 727-735.

Cameron, T. \& Roll, W.G. (1983). An investigation of apparitional experiences. Theta, 11, 74-78.

Claridge, G. (1997). Theoretical background and issues. En G. Claridge (Ed.). Schizotypy: Implications for illness and health (pp. 3-18). Oxford: Oxford University Press.

Claridge, G. \& Beech, T. (1995). Fully and quasi-dimensional constructions of schizotypy. En A. Raine, T. Lencz, y S.A. Mednick (Eds.). Schizotypal personality, 192-216. Cambridge: Cambridge University Press.

Cheyne, J. A.; Newby-Clark, I. R. \& Rueffer, S. D. (1999). Sleep paralysis and associated hypnagogic and hypnopompic experiences. Journal of Sleep Research, 8, 313-317.

Council, J. R. \& Huff, K. (1990). Hypnosis, fantasy activity, and reports of paranormal experiences in high, medium and low fantasizers. British Journal of Experimental and Clinical Hypnosis, 7, 9-13.
Davidson, H. R. E. \& Russell, W. M. S. (Eds.). (1981). The folklore of ghosts. Cambridge, U.K.: Brewer.

Emmons, C. F. (1982). Chinese ghosts and ESP. A Study of paranormal beliefs and experiences. Metuchen, NJ: Scarecrow Press.

Eysenck, H. J. \& Eysenck, S. B. G. (1978). Eysenck personality questionnaire. Madrid: TEA Ediciones.

Finucane, R. C. (1984). Appearances of the dead: A cultural history of ghosts. Búfalo, NY: Prometheus Books.

Fukuda, K.; Ogilvie, R. D.; Chilcott, L.; Vendittelli, A. M. \& Takeuchi, T. (1998). The prevalence of sleep paralysis among Canadian and Japanese college students. Dreaming, 8 , 59-66.

Gauld, A. (1984). Review of Appearances of the dead: A cultural history of ghosts by R. C. Finucane. Journal of the Society for Psychical Research, 52, 330-333.

Green, C. \& McCreery, C. (1975). Apparitions. Nueva York: St Martins Press.

Gómez Montanelli, D. \& Parra, A. (2003). Un abordaje modelo para el procesamiento de las reacciones emocionales ante experiencias paranormales. Revista Argentina de Psicología Paranormal, 14, 9-27. 
Gómez Montanelli, D. \& Parra, A. (2005). ¿Las experiencias paranormales son psicológicamente perturbadoras? Una encuesta comparando estudiantes universitarios y aficionados a temas paranormales. Revista Interamericana de Psicología, 39(2), 285-294.

Grady, K.E. \& Wallston, B. S. (1988). Research in health care setting. Newsbury Park: Sage.

Gurney, E.; Myers, F. W. H. \& Podmore, F. (1886). Phantasms of the living, ( 2 vols). Londres: Trubner.

Hoffman, R. E. (1986). Verbal hallucinations and language production processes in schizophrenia. Behavioral and Brain Sciences, 9, 503-548.

Hufford, D. J. (1982), The terror that comes in the night: An experiencecentered study of Supernatural Assault Traditions. Filadelfia, PA: University of Pennsylvania Press.

Hough, M. (1991). Cognition and the apparitional experience. Australian Parapsychological Review, 17, 8-16.

Irwin, H. J. (1985). Parapsychological phenomena and the absorption domain. Journal of the American Society of Psychical Research, 79, 1-11.

Irwin, H. J. (1989). Extrasensory experiences and the need for absorption. Parapsychology Review, 20(6), 9-10.
James, W. (1902/1958). The varieties of religious experience: A study in human nature. Nueva York: American Library.

Johnson, M. \& Raye, C. (1981). Reality monitoring. Psychological Review, 88, 67-85.

Johnson, M. H.; Hashtroudi, S. \& Lindsay, D. S. (1993). Source monitoring. Psychological Bulletin, 114, $3-28$.

Khor, R. L. (1980). A survey of psi experiences among members of a special population. Journal of the American Society for Psychical Research, 74, 395-411.

Kim, J. \& Mueller, C.W. (1978). Factor analysis. Statistical methods and practical issues. Newsbury Park: Sage

MacKenzie, A. (1982). Hauntings and apparitions. Londres: Paladin.

Matlin, M. W. \& Foley, H. J. (1997). Sensation and perception. Boston: Allyn and Bacon.

Mavromatis, A. (1987). Hypnagogia: The unique state of consciousness between wakefulness and sleep. Londres: Routledge and Kegan Paul.

McCreery, C. (2006). Perception and Hallucination: The case for continuity. Philosophical Paper 2006-1. Oxford: Oxford Forum. 
McCreery, C. \& Green, C. (1986). A follow-up study of people reporting apparitional experiences. Informe presentado en la Décima Conferencia Internacional de la Society for Psychical Research, Cambridge.

McCreery, C. \& Claridge, G. (1995). Out-of-the-body experiences and personality. Journal of the Society for Psychical Research, 60, 129-148.

McCreery, C., \& Claridge, G. (2002). Healthy schizotypy: The case of out-of-the-body experiences. Personality and Individual Differences, 32, 141-154.

Merckelbach, H.; Horselenberg, R. \& Muris, P. (2001). The creative experiences questionnaire (CEQ): A brief self-report measure of fantasy proneness. Personality and Individual Differences, 31, 987-995.

Myers, S. \& Austrin, H. (1985). Distal eidetic technology. Journal of Mental Imagery, 9, 57-66.

Myers, S. A.; Austrin, H. R.; Grisso, J. T. \& Nickelson, R.C. (1983). Personality characteristics as related to the out-of-body experience. Journal of Parapsychology, 47, 131-144.

Ness, R. (1978). The old hag phenomenon as sleep paralysis: A biocultural interpretation. Culture, Medicine and Psychiatry, 2, 15-39.
Osis, K. (1986). Apparitions old and new. En Rao, K. R. (Ed.). Case studies in parapsychology (pp. 74-86). Jefferson, NC: McFarland,

Parra, A.; Adróver, F. \& González, G. (2006). Estudio exploratorio de la experiencia alucinatoria: Comparación entre población clínica y no clínica. En: Trimboli, A.; Fantin, J. C.; Raggi, S.; \& Friedman, J. C. (Eds.). Encrucijadas actuales en salud mental: Primer Congreso Argentino de Salud Mental (pp. 258-267). Buenos Aires, Akadia.

Parra, A. \& Argibay, J. C. (2006). Interacción entre susceptibilidad hipnótica y experiencias disociativas en una población que reporta experiencias anómalo/paranormales. $R e$ vista Interamericana de Psicología, 40, 233-240.

Palmer, J. (1979). A community mail survey of psychic experiences. Journal of the American Society for Psychical Research, 81, 111-123.

Pekala, R. J.; Kumar, V. K. \& Marcano, G. (1995). Anomalous/paranormal experiences, hypnotic susceptibility, and dissociation. Journal of the American Society for Psychical Research, 89, 313-332.

Posey, T. B. \& Losch, M. E. (1983). Auditory hallucinations of hearing voices in 375 normal subjects. Imagination, Cognition and Personality, 2, 99-113. 
Raine, A. (1991). The SPQ: A scale for the assessment of schizotypal personality based on DSM-III-R criteria. Schizophrenia Bulletin, 17, 556-564.

Raine, A. (1992). Sex differences in schizotypal personality in a non-clinical population. Journal of Abnormal Psychology, 101, 361-364.

Raine, A. \& Baker, L. (1992) The Schizotypal Personality Questionnaire: Genetics, Psychophysiology, Neuropsychology and Gender Differences. Portland, Oregon: Western Psychological Association.

Raine, A. \& Benishay, D. (1995). The SPQ-B: A brief screening instrument for schizotypal personality disorder. Journal of Personality Disorders, 9, 346-355.

Richardson, A. (1969). Mental imagery. Nueva York: Springer.

Sarbin, T., \& Juhasz, J. (1967). The historical background of the concept of hallucination. Journal of the History of the Behavioral Sciences, 3, 339-358.

Sartorius, N., Shapiro, R., \& Jablensky, A. (1974). The international pilot study of schizophrenia. Schizophrenia Bulletin, 1, 21-25.

Schneider, K. (1959). Clinical Psychopathology. New York: Grime and Stratton.

Sheehan, P. W. (1967). A shortened form of Betts' questionnaire upon mental imagery. Journal of Clinical Pychology, 23, 386-389.

Sherwood, S. J. (1999). Relationship between childhood hypnagogic hypnopompic and sleep experiences, childhood fantasy proneness, and anomalous experiences and beliefs: An exploratory WWW survey. Journal of the American Society for Psychical Research, 93, 167-197.

Sidgwick, H., Johnson, A., Myers, F.W.H., Podmore, F. \& Sidgwick, E. (1894). Report on the Census of Hallucinations. Proceedings of the Society for Psychical Research, 10, 25-422.

Slade, P. (1976). An investigation of psychological factors involved in the predisposition to auditory hallucinations. Psychological Medicine, 6, 123-132.

Slade, P. D. \& Bentall, R. P. (1988). Sensory deception: A scientific analysis of hallucination. Londres: Croom-Helm.

Smythies, J. (1956). A logical and cultural analysis of hallucinatory sense-experience. Journal of Mental Science, 102, 336-342.

Spanos, N. P.; Cross, P. A.; Dickson, K. \& Dubreuil, S. C. (1993). Close encounters: An examination of UFO experiences. Journal of Abnormal Psychology, 102, 624-32. 
Tart, J. C. (1983a). The controversy about psi: Two psychological theories. Journal of Parapsychology, 46, 313-320.

Tart, J. C. (1983b). Who's afraid of psychic power? Me? The Open Mind, 1(3), 1-5.

Tart, J. C. (1984). Acknowledging and dealing with the fear of psi. Journal of the American Society for Psychical Research, 78, 133-143.

Tellegen, A. \& Atkinson, G. (1974). Openness to absorbing and self-altering experiences ('absorption'), a trait related to hypnotic, susceptibility. Journal of Abnormal Psychology, 83, 268-277.
Thalbourne, M. (1982). A glossary of parapsychology. Londres: Heinemann.

Tien, A. Y. (1991). Distribution of hallucinations in the population. Social Psychiatry and Psychiatric Epidemiology, 26, 287-292.

Wilson, S.C. \& Barber, T.X. (1983). The fantasy-prone personality: Implications for understanding imagery, hypnosis, and parapsychological phenomena. En Sheik, A. A. (Ed.). Imagery: Current theory, research, and applications (pp. 340-387). Nueva York, NY: Wiley. 